\title{
Trowels, processors and misunderstandings: concluding thoughts.
}

\author{
By Mehdi Saqalli, Marc Vander Linden
}

\begin{abstract}
This conclusive text deals with the challenges of interdisciplinarity: Although conceptual and formal thresholds are well known within the scientific community, one may encounter difficulties for answering such related requirements: among them, the tension between the inherent holistic pretence of modelling and datarelated prosaicism is important as well as the simple fact that things, practices, assumptions and words are not the same among disciplines inducing unsolved misconceptions, misunderstandings and social bias. Also, seeking legitimacy and validity but also efficiency at the modelling project level, implying simplifications, interpolations an generalizations, is often in contradiction with the need for legitimacy at the level of each discipline involved. This is due of course to potentially contradictory scientific requirements of both levels and for a weight at least equivalent to the perfectly normal social need for recognition of each researcher, usually within its own discipline, to compare with the risky "business" in terms of reputation, results' over-expectation and time consumption of modelling approaches.
\end{abstract}

\section{Key-words}

Research community; social constraints to interdisciplinarity; go-and-return between fields and models;

\section{Supporting the holistic hope of Archaeology}

Archaeology, under its many theoretical variants, had always had a complex relationship with its selfacknowledged role as the gatekeeper of the past intricacy of all facets of human life over the very longue durée. Having to deal with a record first and foremost characterised by his patchiness and unevenness is to some extent counter-balanced by extraordinary access to an exciting diversity of sources, reflected by the countless methodological and technical refinements of the last few decades. We know that our window unto the past will always remain clouded in much haze, but we are constantly nudging it a bit more open. As a result, and perhaps more so than any other social sciences, most archaeologists - explicitly or not - seem to subscribe to a Zeitgeist dominated by the idea that their discipline's goal is to explore the complexity of cultural and social human life in its entirety while at the same time avoiding the meta-synthesis "original sin", more or less the same positioning as anthropologists for present-time social science.

Well in line with the wider intellectual ethos of their time, foundational texts of what came to be known as processual archaeology are dominated by one of the various versions of system theory (e.g. Doran 1970, Salmon 1978). This trend is most noticeable in David Clarke's contribution with his conceptualisation of several specialised sub-systems all locked in many interaction and feedback loops (Clarke, 1968). 
Regardless of the merits and pitfalls of this approach, it is obvious that its underlying rationale was to provide a - overwhelmingly - formal total view of past human lifeways, a holistic ambition which permeated Clarke's entire oeuvre covering archaeological terminology and even fieldwork strategy (Evans et al. 2006). The holistic aspiration of archaeology is perhaps even more obvious in the following post-processual archaeology, with its constant obsession of all facets of human life permeating one another (e.g. Shanks and Tilley 1987; Hodder 1991). All in all, despite the apparent turbulence of theoretical agendas, a strong case can be made for archaeology constant interest towards all-encompassing approaches, perhaps as reaction to the sketchiness of its empirical foundations.

These two contrasted examples illustrate archaeology's hesitation between reassuring empirism and holistic pretence. Our purpose is not to challenge this pretence, but rather to methodologically and practically support it. This goal indeed allows us to conceptualise in a different way computational modelling. As stated on many occasions in the previous pages, computational models, and especially agent-based models, enable the formal exploration of the interaction between a wide range of parameters. As pointed out in the introduction, the tension between "modelling" and "other" archaeologists does therefore not rest in their objectives, but in the ways and intellectual decisions taken to achieve them. Modelling implies assuming common rules among archaeological sites: a modeller supposes that there are an equivalent set of rules among archaeological sites, even if these rules are adapted to local conditions, while non-modelling archaeologist acknowledges the necessity of a system ruling locally the different elements (architecture, practices, environment, etc.), but keeping variability at the forefront by not presupposing shared common rules. Especially crucial here is the notion, mentioned on previous occasions, of simplicity, or at least the simplification linked to modelling. Whilst a recurring argument against simulation approaches, the modelling community sees it as an imperative requirement. The drawback of such self-imposed formality is however to expose in crude light the assumptions linking the various parameters, thus making such models easily prone to criticisms. It is fair to say that such criticisms, in many instances, actually make a relevant point, in the sense that the modellers perhaps pay too much attention upon the elucidation of the formal relationships between parameters, seen as a way to avoid deterministic accounts whereby a single factor trumps all others. Another implication is the fact that, indeed and especially in the eyes of outsiders, modellers may overlook some considerations in their selection of parameters. This last point is perhaps made most salient by Roux about the choice of morphometric, rather than technical traits of cultural transmission, and also lies at the core of the methodological designs exposed by Saqalli and colleagues, Le Néchet and colleagues, as well as Carrer and colleagues.

\section{A call for interdisciplinary-formalized archaeology modelling}

There is no single, easy answer to the question of how to best fix parameters. Two remarks however come to mind in view of the present contributions:

Firstly, a successful strategy for qualitative assessments in computational modelling lies in the oft-quoted interdisciplinary nature of the enterprise. This integration of specialists from different fields can occur, as shown by many contributions, at various stages of the process, either towards the very beginning of the model design, or later through inclusion / re-assessment of parameters following a first run of simulations. Although the addition and constant re-evaluation of parameters may come at a computational prize (i.e. 
simulations becoming increasingly demanding and thus long to run), the mathematical flexibility of agentbased models provides the required methodological - relative - ease for interdisciplinary work. Beyond these technicalities, it is essential to recognise that interdisciplinarity does not merely translate into the ever addition of parameters, but also comes with what Saqalli and colleagues call sacrifice on the behalf of all partners. For the integration of various parameters to be effective, decisions, including simplifications, have to be made by all contributors. This notion of sacrifice is the key, both for its methodological implications and for its practical, social dimension: any successful interdisciplinary dialogue - and perhaps even so in the case of computational modelling - rests upon hard-fought equality amongst all participants. Archaeologists, ecologists, computer scientists, regardless of each one expertise considered as equal, with the same voice in the process. Here lies the fact that eventually, gentlemen's agreement and a solid argumentation may become the sole legitimacies for selecting and discriminating parameters and factors amongst an interdisciplinary community. This may sound both awkward and obvious, but must be stressed in practical terms.

Secondly, as archaeological data provide an essential resource in the validation of models, at least parts of them must be designated for this validation step and thereby separated from the designing stage of the models. At best, inference from archaeological models can be of use in delineating a parameter space such as a range of values to be explored through simulations, as for instance in Approximate Bayesian Computation approaches. This situation apart, archaeological data may inform decisions regarding the selection of parameters, but cannot play a direct role in the qualitative assessment of these parameters. This point may prove contentious for many archaeologists, for whom data often constitute an ultimate reference, but is imperative to avoid circular arguments.

\section{Main working institutional obstacles beyond the interdisciplinarity mantra}

This ideal interdisciplinary community situation is rarely met: as stated in our introduction, the rationale of this collection of papers stemmed from the recognition that, although computational modelling was arguably more popular than ever in archaeological circles, it still remains a niche activity. We hypothesize that this state of affairs indicates that the positioning of computational modelling within archaeology remains unclear and, more worryingly, that the practical integration of modelling concepts and methods with the archaeological toolkit and archaeological data is not working. While one may consider this situation as the outcome of a series of misunderstandings between modelling and field archaeologists regarding the notions of simplicity, approximation and eventually truth in the epistemological roots of archaeology, we position these misunderstandings into a more practical debate, where global syntheses remain underrated.

Beyond the epistemological limits, the restraining role of several factors ought not to be overlooked, including:

- The discipline-based organization of numerous journals was an initial constraint, though one admittedly positively and rapidly changing;

- As an opposite, one may point out that institutional evaluation, in institutions keeping tenured positions and during application processes, remains segregated between disciplines and even regressing. Even 
more, the repeated call for interdisciplinarity looks much more the same like in other disciplines where interdisciplinarity become pluridisciplinarity, i.e. the juxtaposition of disciplines with no interaction.

- Although the situation varies enormously across countries, in several instance, funding remains allocated on a discipline base. As a consequence, modelling work-packages are often portions of projects but rarely their main goals. Beyond the financial constraints it induces, it means that specialists are focused on delivering their own work-packages and not necessarily ready to invest time in the modelling procedure, let alone to sacrifice the complexity of their topic for what remains a side objective;

- This last point is reinforced by an often misperception encountered by some of us, to perceive computational modelling as some sort of "sub-contracting", merely added to provide "quantitative shine" or "scientific kudos" to a research proposal for instance. It is noteworthy that this situation is hardly new, and that similar concerns have been repeatedly voiced over the years when various archaeological techniques were developed, and then used and abused, before a successful integration became the norm. This had happened as well in other disciplines such as geography and GIS.

As a humoristic conclusion, one may then, borrowing the Kübler-Ross five steps of grief, propose the five steps of modelling adoption in archaeology:

1. Enthusiasm: modelling is seen as panacea, finding the missing data, solving the flaws in hypotheses, provide beautiful illustrations and maps for articles and generate more funds from funding agencies;

2. Denial: modelling is acknowledged to be highly demanding and time-consuming, implies negotiations between scientists and oversimplify;

3. Reject and anger: modelling may even be a risk of overpowering field archaeologists within the discipline due to this "quantitative shining scientifically trendy kudos";

4. Bargaining: modelling is only a support for improving the process formalizing, combining and deducing archaeological reasoning. Once used properly, it is a powerful weapon for facilitating research, both facing funding agencies and reluctant institutes;

5. Cold acceptance: multidisciplinary project teams slowly self-impose themselves thanks to non-niche funding practices;

We hypothesize that we all pass the phase 1 reaching the phase 2 and even the phase 3 quite abruptly and we are observing in most cases goes-and-returns between phases 2 and 3 in one hand and phase 4 on the other hand with the latter being obviously much more rewarding.

\section{Qualitative factors in modelling}

\subsection{Struggling with qualitative factors}

In 2000, the modeller and sociologist Edmund Chattoe-Brown asked, during the $24^{\text {th }}$ International Conference of Agricultural Economists (IAAE) "Why is building Multi-Agent Models of social systems so difficult? Nearly two decades later, the chapters presented here did not shy away from the complexity encountered in building models integrating qualitative factors. Some appear more at first sight to be relative failures, such as Saqalli and colleagues, Barceló and colleagues and Bentley and O'Brien, while Le Néchet and colleagues discuss the obstacles encountered during the modelling process. 
These apparent failures and struggles underline the inherent difficulty in tackling qualitative socioanthropological factors independently from time (this difficulty concerns simulations of both past and presenttime societies) and tools (the difficulty can be felt with or without computational modelling) and stress the necessity to address the fundamental issue of the adequation between archaeological data and the requirements of modelling and statistical approaches.

As a result, since qualitative factors have by definition, and regardless of modelling, to be considered in any future interpretation of past societies, these difficulties may well be simply due to the fact that, after all, we are still in the early stages of formalizing qualitative factors. However, such integration implies solving or at least improving the methodological formalization of qualitative factors. This challenge is essential in both modelling and, beyond, in any interpretative system as, in all instances, priorities regarding the inclusion or exclusion of factors should be made explicit.

\subsection{Balancing between approaches for KISS and KIDS for modeling RSES}

Therefore, although qualitative factors may in many cases be partly dependent upon biophysical constraints, ethnography and anthropology demonstrate the huge variety of social combinations possible for each biophysical environment. Given that, by definition, it remains impossible, if only from a practical point of view, to offer any total description of any society and its biophysical environments, the selection of factors to be considered remains a crucial, though perhaps somehow overlooked, intellectual task. From this point of view, and going back to the KIDS vs. KISS opposition (Edmonds and Moss 2005), one should clarify when it is relevant to:

- Work on a society as a whole implies an empirical "KIDS" approach (Keep It Descriptive, Stupid"): it sometimes leads to an inflation of factors included, and results in fuzzy results. Such models often require the integration of parameters for which direct analogues are either lacking or extremely remote, thus lowering the quality requirements of their integration. As a result, KIDS can never be complete, meaning they cannot cover and model all aspects of past social life. A selection of main issues is to be assessed;

- Focus on one social phenomenon, implying a possibly more operative and straightforward "KISS" approach (Keep It Simple, Stupid"): whilst it possibly leads to apparently more precise results, this approach requires explicit justification of the choice and the relevance of the limited sets of parameters exposed, unless simulating dynamics that never occurred in reality, overpowered by neglected and nonsimulated factors. As a result, KISS can never be exact, meaning they can never completely explain the priority given to the chosen dynamics.

Combining these elements are then necessary and we support the acknowledgement that modelling the past is always a balance between KIDS and KISS extreme poles, as a medium way for simulating Rural SocioEcological Systems (RSES). This balance should be always justified according to the importance of the selected dynamic to simulate. 


\subsection{Beyond Socio-ecological Systems}

Indeed, regarding RSES, biophysical parameters are positive constraints that both increase local conditionalities, reducing the dispersal of social hypotheses but also provide testing data for validating purposes, giving more confidence to modelling approaches. We then here question the challenge of modelling factors and societies that are poorly conditioned by such biophysical factors, such as norms rules and customs, non-farming social classes and urban societies. That would be the next challenge for modelling archaeologists. A possible way is to focus on ceteris paribus situations where other factors apart from the studied social issues remain or can be assumed as stable. It means simulating over long but very stable periods of time, over short periods of time or even over very short periods with only the studied dynamic.

As repeatedly said by Charles Darwin, "one cannot be a good observer without being at the same time an active theorist". A good archaeologist has to be also a conceptualist and modelling should then be seen as the support for this demarche balancing between field and formalization. One may then consider that the future of archaeology will increasingly include modelling tools in the many historical cases where biophysical constraints failed to be sufficient enough for explaining past population dynamics.

\section{References}

Clarke D. 1968. Analytical archaeology. Methuen, London, UK.

Doran J. 1970. Systems theory, computer simulations and archaeology. World Archaeology, 1, 3, 289-298.

Evans C., Edmonds M., Boreham S., Evans J., Jones G., Knight M., Legge T. 2006. 'Total archaeology' and model landscapes: excavation of the great Wilbraham causewayed enclosure, Cambridgeshire, 1975-76. Proceedings of the Prehistoric Society, 72: 113-162. doi:10.1017/S0079497X00000803

Hodder I. 1991. Reading the past: current approaches to interpretation in archaeology.: Cambridge University Press, Cambridge, UK.

Salmon M. H. 1978. What can systems theory do for archaeology? American Antiquity 43, 2, 174-183.

Shanks M. Tilley C. 1987. Re-constructing archaeology: theory and practice. Routledge, London, UK.

Chattoe E. 2000. Why is building Multi-Agent Models of social systems so difficult? A case study of innovation diffusion. 24th International Conference of Agricultural Economists (IAAE).

Edmonds B., Moss, S. 2005. From KISS to KIDS: an "anti-simplistic" modelling approach. Lecture Notes in Artificial Intelligence 34, 130-144.

Moss S., Edmonds B. 2005a. Towards good social Science. JASSS 8, 13.

Moss S., Edmonds B. 2005b. Sociology and simulation: statistical and qualitative cross-validation. Am. J. Soc. 110, 1095-1131. 